\title{
MECHANICAL AND STRUCTURAL PROPERTIES OF COLLAGEN NANOFRIBROUS LAYERS UNDER SIMULATED BODY CONDITIONS
}

\author{
Jitka Říhová ${ }^{a, *}$, Tomáš SuchÝ ${ }^{b}$, Lucie Vištejnová ${ }^{c}$, LukÁŠ HornÝa ${ }^{a}$, \\ MONIKA ŠUPOVÁ ${ }^{b}$ \\ ${ }^{a}$ Czech Technical University in Prague, Faculty of Mechanical Engineering, Department of Mechanics, \\ Biomechanics and Mechatronics, Technická 4, 16607 Prague 6, Czech Republic \\ ${ }^{b}$ Czech Academy of Sciences, Institute of Rock Structure and Mechanics, V Holešovičkách 94/41, 18209 Prague \\ 8, Czech Republic \\ ${ }^{c}$ Charles University, Medical Faculty in Pilsen, Biomedical Center, alej Svobody 1655/76, 32300 Pilsen, Czech \\ Republic \\ * corresponding author: jitka.rihova@fs.cvut.cz
}

\begin{abstract}
The theme of this paper is the analysis of mechanical and structural properties of nanofibrous COL under simulated body conditions and in the presence of osteoblasts and dermal fibroblasts. COL were prepared by electrostatic spinning of $8 \mathrm{wt} \%$ collagen type I dispersion with $8 \mathrm{wt} \%$ (to COL) of PEG in phosphate buffer/ethanol solution (1/1 vol). The stability of COL was enhanced by means of cross-linking with EDC and NHS at a molar ratio of 4:1. COL were exposed in culture medium for 21 days and human SAOS-2 human dermal fibroblasts and osteoblasts were cultured therein for 21 days as well.

The cell culture on COL was assessed by fluorescence microscopy and metabolic activity. Then the metabolic activity of both cell types grown on COL and PS were measured after 1, 7, 14 and 21 days using the Alamar Blue assay method. Mechanical properties were determined using an tensile test. The influence of the cell activity on secondary structure of COL was verified by IR spectroscopy. Furthermore, the influence of cells on COL was evaluated by SEM.
\end{abstract}

KEYWORDS: Collagen, fibroblasts, osteoblasts, mechanical properties, structural properties, biodegradation.

\section{INTRODUCTION}

Part of the tissue engineering is research and development of new materials which will be used in biomedicine. One of the directions is the development of biocompatible nanofibers which can be prepared, for example, from polymers. These nanostructures can provide temporary mechanical and structural support for cells and help restore damaged tissue. Current research in the field are concentrated mostly on materials that are completely resorbable in the human body over time and their properties can simulate the function of the original tissue.

Understanding the factors that influence their degradation is crucial for the development of polymer degradable systems, especially in terms of degradation kinetics, changes in mechanical properties, and identification of degradation products [1, 2]. The term biodegradation has been known for degradation occurring in biological environment. In the context of biomedical applications, biodegradation can be defined as the gradual decay of a material driven by a specific biological activity [3, 4, Changes in the physico-chemical properties of materials that are exposed to body fluids are the result of chemical, physical, mechanical, and biological interactions between the material and the environment [5, 6].

\section{MATERIAL AND METHODS}

\subsection{NANOLAYER PREPARATION}

Collagen nanolayers (COL) were prepared by electrostatic spinning (4SPIN, Contipro, Czech Republic). The first step in the preparation was to mix the desired amount of collagen type I (calf skin, VUP Medical, Czech Republic) with polyethylene oxide (Mw 900,000, Sigma-Aldrich, Germany) and phosphate buffer (PBS, Sigma-Aldrich, Germany). This mixture was incubated at $37^{\circ} \mathrm{C}$ for 3 days. The hydrolysed collagen must be homogenized (10000 rpm, T25, Ultra-Turrax, IKA, Germany) to form a collagen dispersion, to which ethanol (Penta, Czech Republic) is added. The electrospinning solution is prepared as $8 \% \mathrm{w} / \mathrm{w}$. collagen in a mixture of phosphate buffer and ethanol (1:1 by volume). The proportion of polyethylene oxide, as a auxiliary polymer which was completely washed after crosslinking of the nanofiller, was $8 \%$ by weight (to collagen).

After that COL had to be cross-linked, which helps to increase its stability. The crosslinking was performed chemically with $95 \%$ ethanol (Penta, Czech Republic) with N-(3-dimethylaminopropyl)N-ethylcarbodiimide hydrochloride (EDC, SigmaAldrich, Germany) and N-hydroxysuccinimide (NHS, 
Sigma-Aldrich, Germany). The EDC / NHS molar ratio was $4: 1$. In this solution, the nanoparticles were soaked for 24 hours at $37^{\circ} \mathrm{C}$. Finally, the COL is rinsed with sodium phosphate (Penta, Czech Republic) three times for 20 minutes. Subsequently, the COL were twice rinsed in distilled water, which is important for the complete washing of the polyethylene oxide. Finally, washed COL were frozen $\left(-30^{\circ} \mathrm{C}\right)$ and dried in a lyophilizer (VirTris Benchtop, USA) [7].

\subsection{EXPOSURE IN CULTURE MEDIUM AND IN THE PRESENCE OF CELLS}

For the exposure of COL in simulated body conditions, dermal fibroblasts and osteoblasts (human line SAOS-2) cultivated on the surface of the layers were used. Both cell types were first cultivated on $75 \mathrm{~cm}^{2}$ polystyrene culture bottles (Techno Plastic Products, Switzerland) in culture medium. The culture medium contained Dulbecco's Modified Eagle Medium (DMEM, Thermo Fisher Scientific, USA), fetal bovine serum (FBS, Thermo Fisher Scientific, USA), penicillin $(100 \mathrm{U} / \mathrm{ml})$, streptomycin $(0.1 \mathrm{mg} / \mathrm{ml})$, L- glutamine, and $1 \%$ non-essential amino acid (Biosera, USA). Cultivation took place at $37^{\circ} \mathrm{C}$ and $5 \% \mathrm{CO} 2$. On the day of the experiment, the dermal fibroblasts were sedimented with trypsin/EDTA and counted using a Bürker cell. For fluorescence microscopy and for measuring metabolic activity, COL were prepared in the form of a $0.3 \mathrm{~cm}$ circle, rinsed for $20 \mathrm{~min}$ in $1 \%$ ethanol and $10 \mathrm{~min}$ in PBS. The samples were then placed in a 96 polystyrene panel (Techno Plastic Products, Switzerland) and immersed in $150 \mu \mathrm{m}$ of culture medium until they were seeded with cells.

For mechanical tests, $9 \times 50 \mathrm{~mm}$ strips were rinsed for 20 minutes in $70 \%$ ethanol and 10 minutes in PBS. $20 \times 50 \mathrm{~mm}$ (SPL Life Sciences, Korea) culture chambers were each placed in two pieces and immersed in $4 \mathrm{ml}$ of culture medium until they were fitted with cells. The dermal fibroblasts were diluted to a culture medium at a concentration of 30,000 cells $/ \mathrm{ml}$ and loaded onto collagen nanoparticles at a deposition density of 15,000 cells $/ \mathrm{cm}^{2}$ and a volume of $150 \mu \mathrm{l}$. The osteoblasts were diluted to a culture medium at 30,000 cells $/ \mathrm{ml}$ and loaded onto collagen materials at a loading density of 15,000 cells $/ \mathrm{cm}^{2}$ and a volume of $150 \mu \mathrm{l}$. Both cell types were cultured for 21 days, when the measurements ranged on days 1, 7, 14 and 21. The culture medium was changed three times a week.

\subsection{BiologicAl tests}

The cell culture on COL were assessed by fluorescence microscopy and metabolic activity. First, the proper seeding by both cell types was evaluated. Dermal fibroblasts and osteoblasts were dyed at 1, 7, 14 and 21 days after the start of the experiment. At each time, both cell types were cultured on COL and on polystyrene (PS) stained with a $1 \mu \mathrm{g} / \mathrm{ml}$ solution of calcein AM (Thermo Fisher Scientific, USA) in culture medium for $30 \mathrm{~min}$ at $37^{\circ} \mathrm{C}$ and $5 \% \mathrm{CO}$. After the staining period, the samples were rinsed with PBS and transferred to the cover glass. Clear fluorescence images of both types of cells cultured on COL and PS were acquired using the Olympus IX83 (Olympus, Switzerland) microscope at $488 \mathrm{~nm}$ excitation and $528 \mathrm{~nm}$ emission. Images were processed using ImageJ (National Institute of Health, Bethesda, USA).

The metabolic activity of dermal fibroblasts and osteoblasts grown on COL and PS were measured at 1, 7, 14 and 21 days after the start of the experiment using the Alamar Blue assay method. From each sample, culture medium was aspirated and $150 \mu \mathrm{l}$ of new culture medium containing $10 \times$ diluted Alamar Blue (ThermoFisher Scientific, USA) was added. Samples were incubated for 2 hours at $37^{\circ} \mathrm{C}$ and $5 \% \mathrm{CO} 2$. Subsequently, $100 \mu \mathrm{l}$ of culture medium was pipetted into a 96 plate (NUNC, Denmark) and the fluorescence of the solution at $530 \mathrm{~nm}$ excitation and $590 \mathrm{~nm}$ emission was measured using a Synergy HT (Biotek, USA) spectrophotometer. From the measured values, the fluorescence of the culture medium itself was read and the values were expressed as the fluorescence ratio at day 7, 14 and 21 on fluorescence. Values thus reflect the change in the metabolic activity of cultured cells on COL and on PS over time. The experiment was performed in 6 replicates.

\subsection{Mechanical tests}

Changes in mechanical properties were measured by a uniaxial tensile test in which the initial modulus of elasticity in the first and the last (fifth) load cycle and the ultimate tensile strength of the collagen layer were evaluated. The tensile test was carried out in the laboratory of biomechanics at the CTU in Prague. Measurements were carried out on a multi-axis test machine (Zwick/Roell, Germany) with force sensors U9B by HBM with a range of $\pm 25 \mathrm{~N}$ and $\pm 250 \mathrm{~N}$ equipped with a non-contact optical extensometer. This extensometer, using the contrast marks on the samples, records the course of deformation during loading. The data was recorded at a frequency of $20 \mathrm{~Hz}$ and stored on a computer. The position of the marks, the position of the clamping jaws and the force were measured. Mechanical tests were performed for groups of 8 samples in medium, with osteoblasts and fibroblasts for each time interval. Because of viscoelastic behavior of hydrated COL, which also depends on the previous deformation, it is necessary to load and relieve the sample several times before performing the inter-strength test. Estimation of the initial modulus of elasticity occurred both for the first load cycle, thanks to which we can determine the effect of dermal fibroblasts and osteoblasts on the surface layers of the material, as well as the last load cycle from which the effect of cells on the inner structure of the material can be estimated. From the measured data, graphs of stress $\sigma$ - strain $\epsilon$ relationships were plotted. The values for these charts were calculated 
from:

$$
\begin{gathered}
\sigma=\frac{F}{S} \quad[\mathrm{MPa}], \\
\epsilon=\frac{\Delta l}{l_{0}} \quad[-],
\end{gathered}
$$

where $\mathrm{F}$ is the force measured by the force sensors $[\mathrm{N}], \mathrm{S}$ is the cross sectional area of the unloaded sample $\left[\mathrm{mm}^{2}\right]$ (we consider the rectangular cross-section), $\Delta l$ is the elongation of the sample determined by the change of the contrast marks on the samples [mm] and $l_{0}$ is the initial length of the sample [mm].

\subsection{Structural Changes}

Structural changes were monitored by infrared spectroscopy and scanning electron microscopy. Secondary structure of COL was studied by infrared spectrometry (FTIR), which allows study of its changes (denaturation) after various processes (isolation, crosslinking, sterilization). The aim of this analysis was to verify the possible effect of cell activity as well as exposure in the culture medium to the secondary collagen structure. The structure of COL was studied using Protégé 460 E.S.P. (Thermo Nicolet Instruments, USA) by a method of attenuated total reflection ATR (GladiATR, PIKE Technologies, USA) with a diamond crystal. The resulting spectra were obtained from 128 scans with a resolution of $4 \mathrm{~cm}^{-1}$. Belt areas (integral absorbance) were determined using OMNIC 7 software, which was also used for the deconvolution procedure of the amide bands I. The input data for band separation (number, position, half width, relative intensity and shape) were obtained using the Fourier self-deconvolution procedure and further specified by the fitting procedure using the Gaussian profile function. The individual COL were measured at 10 different sites and the values of the waist areas after deconvolution were statistically evaluated. A SEM analysis was used to basically assess the surface of collagen layers Quanta 450 electron microscope (FEI, USA). To assess the effect of cultivation on the surface of COL the images (magnification $500 \times$ and $25,000 \times$ ) were taken at time intervals on day 1 , day 7 , day 14 , and day 21. Another reason of the SEM analysis was to determine the fiber diameter before and after the crosslinking. To evaluate these data, the diameter of the fibers was evaluated manually by measuring their diameter (at magnification of $25,000 \times$ ). All samples were sputter-coated with a thin layer of gold (Emissions K550X, Quorum Technologies, UK) prior to scanning.

\section{Results}

\subsection{BiologicAl test}

After the first day of culture, both dermal fibroblasts and osteoblasts were similar to the rounded shape of the cell on PS and on the COL (see Fig. 1). In the following days of cultivation $\left(7^{\text {th }}, 14^{\text {th }}, 21^{\text {st }}\right.$ day) dermal fibroblasts on PS showed a typical elongated cell shape and covered most of the area planted. Most cells were oriented in the same direction. In contrast, dermal fibroblasts on COL did not show a one-way arrangement. The cells had a typical shape with elongated protrusions throughout the COL space indicating their physiological comfort. On the $7^{\text {th }}, 14^{\text {th }}$, and $21^{\text {st }}$ days of cultivation, osteoblasts on PS showed a typical cuboid shape and covered most of the area planted. Osteoblasts on COL were more rounded, and the cells grew on multiple layers (see Fig. 1).
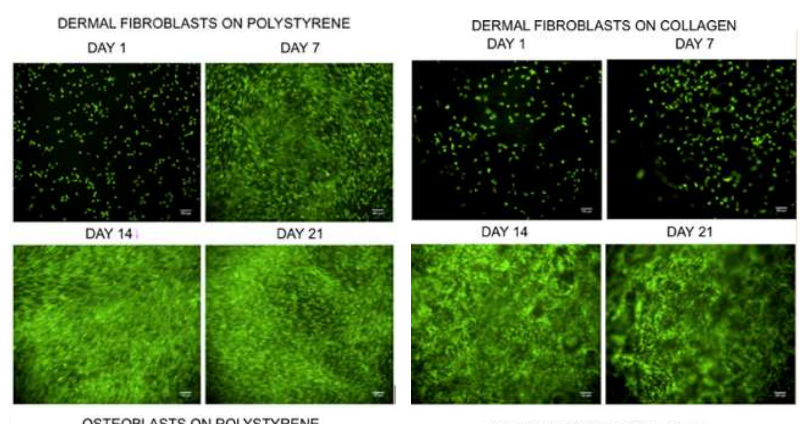

DAY 1
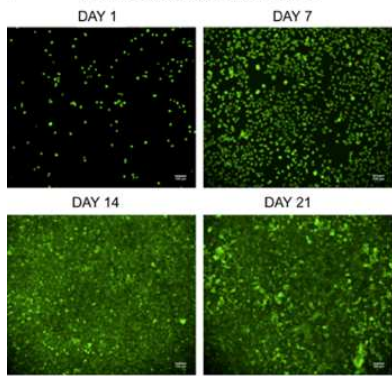

OSTEOBLASTS ON COLLAGEN
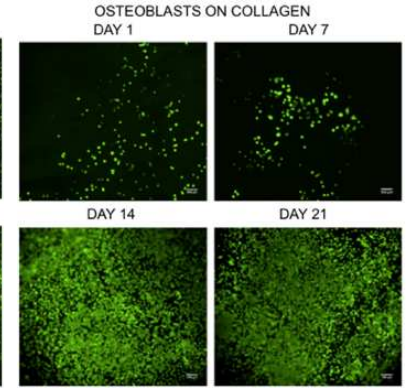

Figure 1. Dermal fibroblasts and osteoblasts cultivated on polystyrene and collagen were stained with calcein AM solution, green color signifies metabolically active cells (scale $100 \mu \mathrm{m}$ ).

Compared to the first day, the metabolic activity of dermal fibroblasts on the COL was 9 times higher $(8.8 \pm 2.5)$ after 7 days of culture, and 5 times higher on PS $(4.5 \pm 0.6)$. This difference between COL and PS was statistically significant $(p<0.05)$. After 14 and 21 days of culture, the metabolic activity of these cells on the COL was 6 times higher $(5 . \pm 0.7$ and $5.9 \pm 1.4)$. On PS after 14 days 6 times and 21 days 5 times higher $(5.6 \pm 0.7$ and $5.3 \pm 0.4)$ versus the first day. These differences between COL and PS were not statistically significant $(p<0.05)$ (see Fig. 2). Metabolic activity of osteoblasts was $10 \mathrm{x}$ higher $(9.7 \pm 2.9)$ on the COL after 7 days of cultivation, and $11 \times$ higher $(11.0 \pm 1.6)$ on the PS compared to the first day. This difference was not statistically significant $(p<0.05)$. After 14 days of cultivation, osteoblastic metabolic activity on COL was 7 times higher $(7.3 \pm 0.5)$ and on PS nearly 13 times higher $(12.7 \pm 1.8)$ versus day 1 . After 21 days, their metabolic activity on COL $10 \times$ higher $(10.2 \pm$ $3.5)$ and on PS nearly 16 times higher $(15.8 \pm 0.9)$. Differences between COL and PS were statistically significant $(p<0.05)$ at both time points (see Fig. 2). 


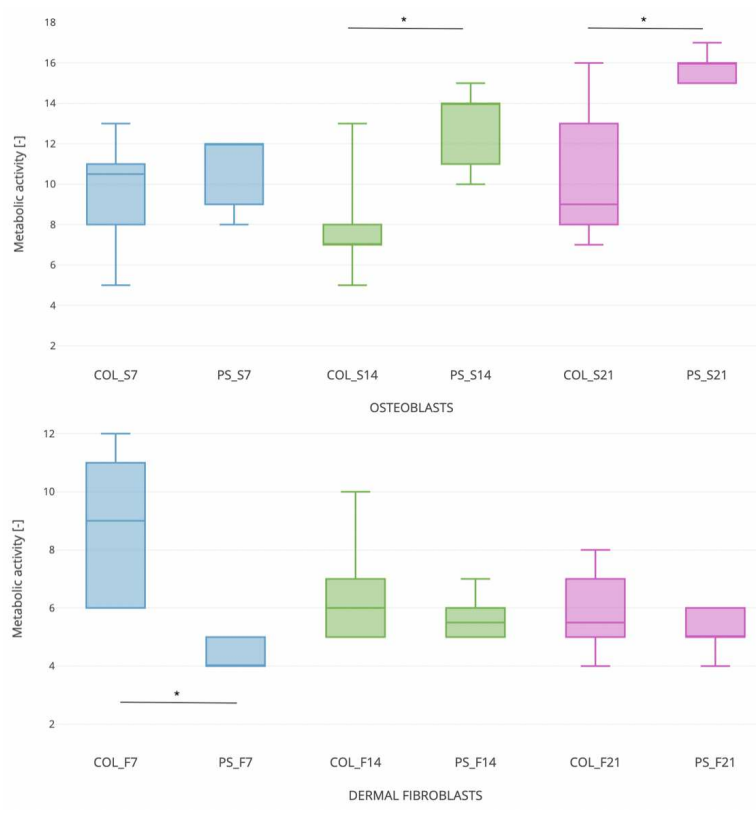

FiguRE 2. Metabolic activity of dermal fibroblasts and osteoblasts grown on COL and PS, * indicates statistically significant differences $(p=0.05$, t-test)

\subsection{Mechanical properties}

From the measured data on samples exposed to medium without or with both cell types $(n=8)$ on the $1^{\text {st }}, 7^{\text {th }}, 14^{\text {th }}$ and $21^{\text {st }}$ days, stress-strain relationships were plotted in Figs. 34 . From the measured data, the initial modulus of elasticity was obtained as the slope of the tangent made to a stress-strain relationship on the initial linear part in the first and the last cycle. The ultimate tensile strength was determined as the maximum observed value of the nominal stress during the last loading cycle sample. The samples were labeled as follows: $M$ for samples exposed only in medium, F for samples with dermal fibroblasts and $\mathrm{S}$ for samples with osteoblasts.

The values of the initial elastic moduli in the first load cycle were not different for the samples exposed on the $1^{\text {st }}$ and $21^{\text {st }}$ day (see Fig. 5. In both dermal fibroblasts and osteoblasts (see Fig. 5), the values of the initial modulus of elasticity in the first loading cycle were decreased by $16 \%$ and $36 \%$. A statistically significant difference in the last load cycle was observed only in osteoblast specimens (see Fig. 5). For samples in the medium and fibroblast samples (see Fig. 5), the difference in values was not statistically significant. For samples exposed in the medium, fibroblast samples and osteoblast samples, a statistically significant increase in the strength limit was recorded, by $12 \%$ (see Fig. 6).

\subsection{Structural properties}

\subsubsection{INFRARED SPECTROSCOPY}

Collagenous materials were comparable from a spectroscopic point of view. The quartet of the $\sim$ $1205,1240,1280$ (amide III) and $1340 \mathrm{~cm}^{-1}$ quartets

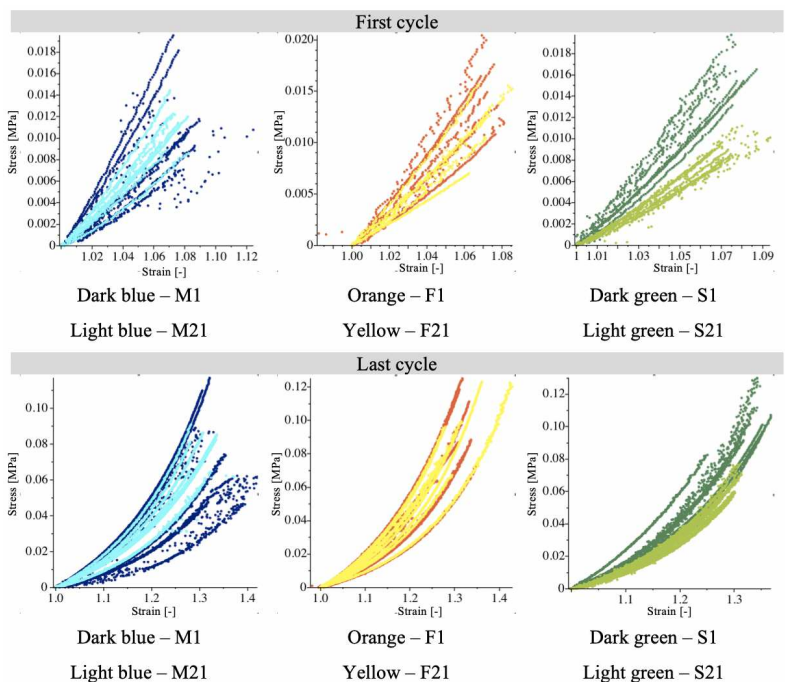

FigURE 3. Stress-strain graphs for the first and last load cycles. For specimens exposed in 1. (M1) and 21 (M21), samples with fibroblasts (F1) and day 21 (F21) and samples with osteoblasts (S1) and day 21 (S21).
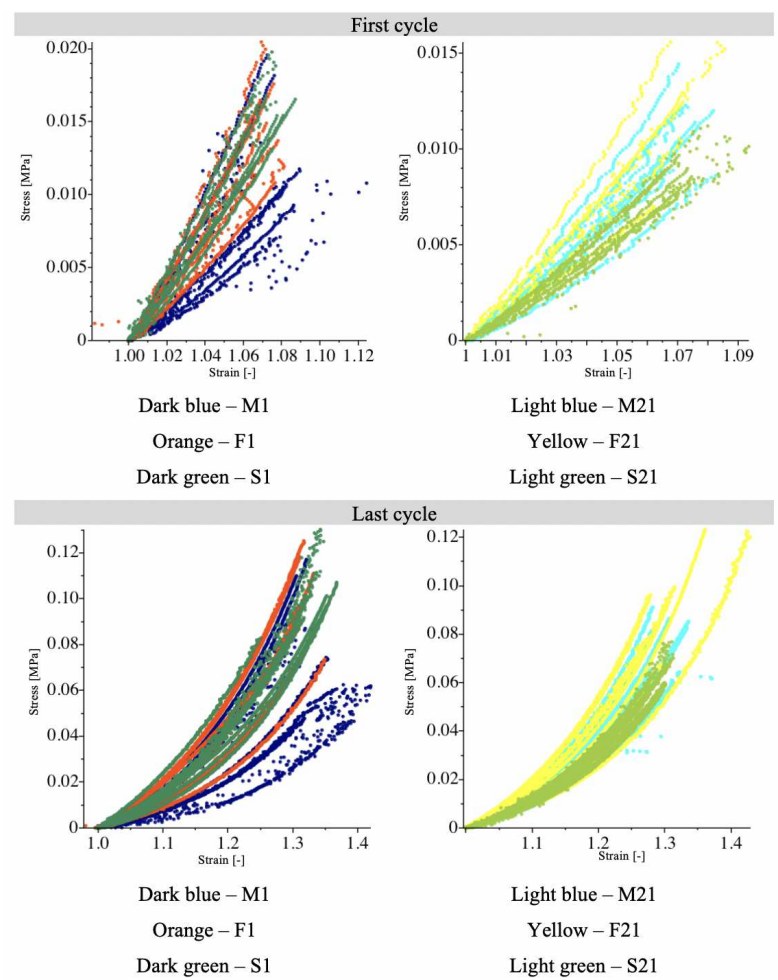

Figure 4. Stress-strain graphs for the first and last load cycles. For specimens exposed in 1. (M1) and 21 (M21), samples with fibroblasts (F1) and day 21 (F21) and samples with osteoblasts (S1) and day 21 (S21).

were a clear proof of the existence of a helical structure, in other words, the natural collagen structure was maintained at the appropriate level during the preparation of the layers by electrostatic spinning, to its fundamental violation. Spectral decomposition of the amide band I made it possible to determine the ratio of the helical portion of collagen represented by the $1660 \mathrm{~cm}^{-1}$ band to the denatured structures based 


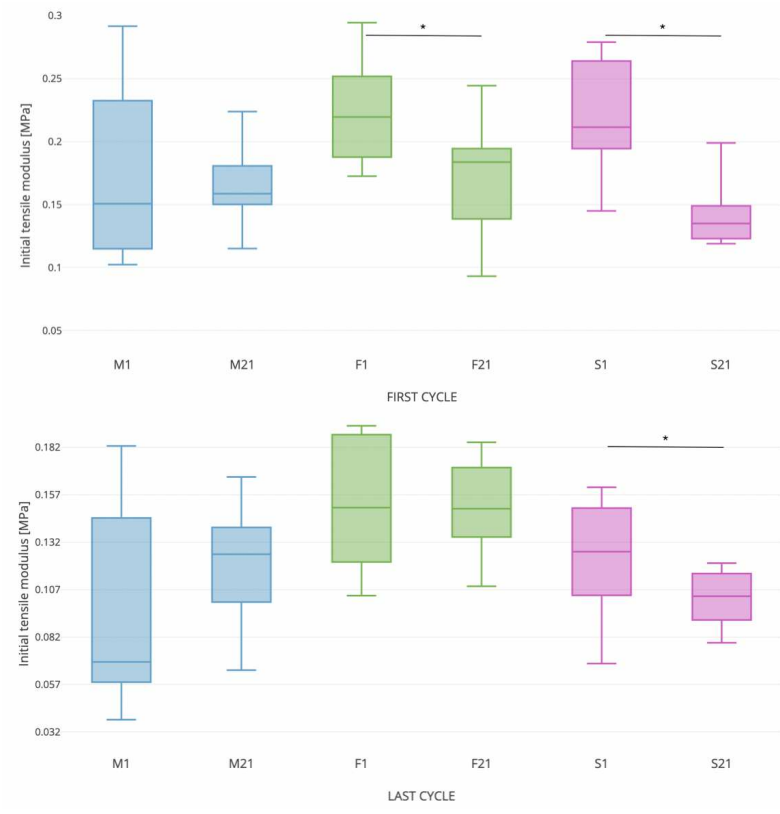

Figure 5. Box plot comparing the initial tensile modulus in the first and the last cycle 1. day (M1) and 21. (M21) day of samples exposed in medium, 1. (F1) and 21. day (F21) of fibroblast, 1. (S1) and 21 (S21) of osteoblast samples, * indicates statistically significant differences (Mann-Whitney, 0.05)

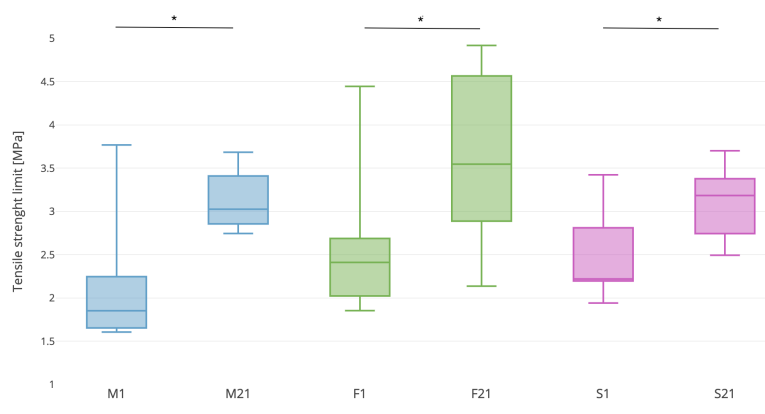

Figure 6. Box plot comparing the tensile strength limit of 1 (M1) and 21 (M21) of the day of samples exposed in medium, F1 and F21 of Fibroblast samples, 1 (S1) and 21. day (S21) of osteoblast specimens, * indicates statistically significant differences (MannWhitney, 0.05)

on the $1630 \mathrm{~cm}^{-1}$ bands and the $1615 \mathrm{~cm}^{-1}$ band.

The $1660 /(1630+1615)$ ratios were determined from measurements of 10 different sites of the collagen layers exposed to the culture medium (M1 and M21) and of the layers exposed to the cells. Fibroblasts (F1 and F21) and osteoblasts (S1 and S21). The obtained data were statistically evaluated and compared. As can be seen, only the S1 and S21 pairs are statistically significant (see Fig. 7).

\subsubsection{ELECTRON MICROSCOPY}

The average fiber diameter of the sample before crosslinking was $261 \pm 49 \mathrm{~nm}$. The average fiber diameter of the sample after crosslinking was $260 \pm 35 \mathrm{~nm}$. As can be seen from Fig. 8 the fiber diameters before and after

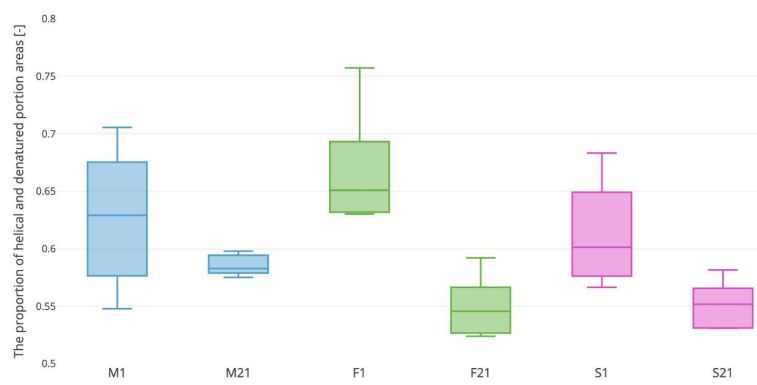

Figure 7. Box plot comparing ratio of helical and denatured areas of collagen $(1660 /(1630+1615)) 1$. day (M1) and 21. (M21) day of samples exposed in medium, 1. (F1) and 21. day (F21) of fibroblast, 1. (S1) and 21 (S21) of osteoblast samples

the cross-linking were not statistically significantly different.

The mean fiber diameter of the sample did not changed during the exposure in the medium (202 \pm $30 \mathrm{~nm}$ on the first day and $196 \pm 26 \mathrm{~nm}$ on the last day). The mean fiber diameter of the fibroblast samples after cross-linking the first day was $187 \pm 33 \mathrm{~nm}$, the last day $184 \pm 32 \mathrm{~nm}$. For osteoblast samples the mean fiber diameter of the after crosslinking was $198 \pm 27$ on the first day and $198 \pm 25$ on the last day. From Fig. 9 it was obvious that the fiber diameter values were not statistically significant in the case of exposed samples.
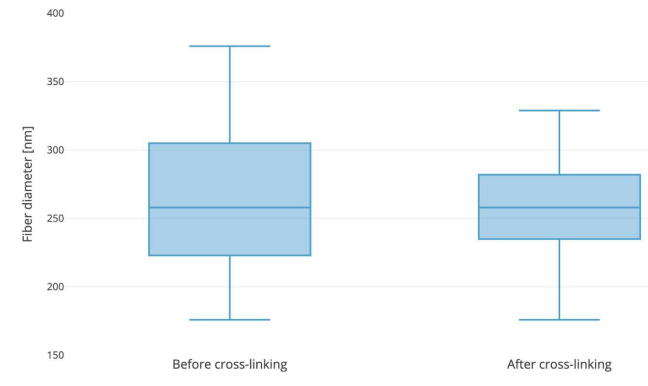

FiguRE 8. A box plot comparing the fiber diameter before and after the crosslinking. Fiber diameters are not statistically significant different.

\section{Conclusion}

During this work it was found that both fibroblasts and osteoblasts can influence the mechanical and structural properties of collagen layers by their activities. The activity of the cells has been demonstrated especially in the case of osteoblasts. In their presence there was a reduction in the modulus of tensile elasticity, which manifested itself in both the first and last load cycles. In the case of fibroblasts, this phenomenon was observed only in the first cycle, ie in a situation where the mechanical properties are more affected by the properties of the surface layers. In the case of an increase in the tensile strength after a three-week exposure, it was not shown that it was due to cell 


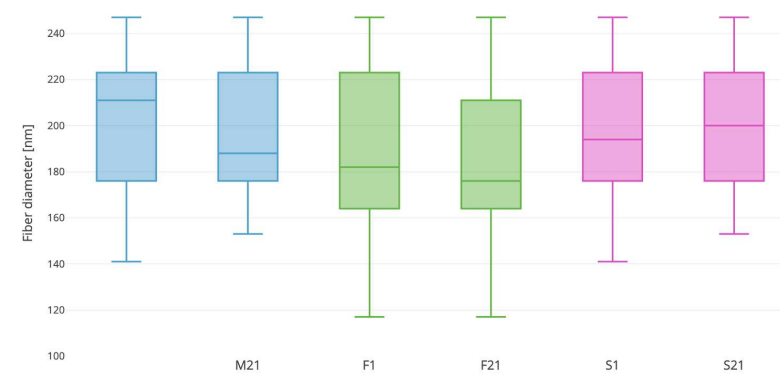

FiguRE 9. A box plot comparing the fiber diameter after first (M1) and 21 (M21) in medium, 1. (F1) and 21. (F21) days of fibroblast culture and 1. (S1) and 21. (F21) days of culture of fibroblasts and 1. (S1) and 21. (S21) days of cultivation with osteoblasts. Fiber diameters are not statistically significant different.

activity, since higher strengths were also achieved in samples exposed in the cell-free medium itself. Higher activity of osteoblasts was further demonstrated by infrared spectroscopy, which showed an increase in the ratio of helical collagen to denatured structures. The presence of cells did not affect the diameter of the fibers of the collagen nanoparticle.

Fibroblasts and osteoblasts were succesfully plated and cultured on COL. Changes in the initial modulus of elasticity were observed most in the first load cycle, where both the fibroblast and the osteoblast samples showed statistically significant differences between the $1^{\text {st }}$ and $21^{\text {st }}$ day of the cultivation. For the last load cycle, statistically significant changes between $1^{\text {st }}$ and $21^{\text {st }}$ day of cultivation were observed only in samples with osteoblasts. On the other hand, ultimate tensile strenght increased in both cell culture media as well as in layers with cultured cells, thus the changes in ultimate tensile strength cannot be addressed only to cell activity. The infrared spectroscopy revealed the changes in helical structure of COL only in osteoblast specimens. Fiber diameters changes which were measured from images from electron microscopy were not observed.

Fibroblasts and osteoblasts can influence mechanical and structural properties of collagen nanoparticles. Identification of cellular mechanisms responsible for the mechanical changes will be the aim of following research focused on the analysis of enzymatic activity and remodelation procedures of dermal fibroblasts and osteoblasts.

\section{ACKNOWLEDGEMENTS}

The study was supported by project No. CZ.02.1.01/0.0/0.0/16_019/0000787 "Fighting INfectious Diseases", awarded by the Ministry of Education, Youth and Sports of the Czech Republic, financed from The European Regional Development Fund.

\section{REFERENCES}

[1] K. Jahan, M. Tabrizian. Composite biopolymers for bone regeneration enhancement in bony defects. Biomaterials Science 4(1):25-39, 2016. DOI:10.1039/c5bm00163c

[2] F. O'Brien. Biomaterials \& scaffolds for tissue engineering. Materials Today 14(3):88-95, 2011. DOI:10.1016/S1369-7021(11)70058-X

[3] H. Azevedo, R. Reis. Understanding the Enzymatic Degradation of Biodegradable Polymers and Strategies to Control their Degradation Rate. CRC Press, Boca Raton, 2005. DOI:0.1201/9780203491232.ch12

[4] L. Ruiz-Rubio, L. Perez-Alvarez, J. Vilas-Vilela. Biodegradable shape-memory polymers. Advanced Structured Materials 115:219-236, 2020. DOI:10.1007/978-981-13-8574-2_10

[5] D. Williams, S. Zhong. Biodeterioration/biodegradation of polymeric medical devices in situ. International Biodeterioration and Biodegradation 34(2):95-130, 1994. DOI:10.1016/0964-8305(94)90002-7

[6] D. Kytyr, P. Zlamal, P. Koudelka, et al. Deformation analysis of gellan-gum based bone scaffold using on-the-fly tomography. Materials and Design 134:400-417, 2017. DOI:10.1016/j.matdes.2017.08.036

[7] T. Suchy, M. Supova, E. Klapkova, et al. The release kinetics, antimicrobial activity and cytocompatibility of differently prepared collagen/hydroxyapatite/vancomycin layers: Microstructure vs. nanostructure. European Journal of Pharmaceutical Sciences 100:219-229, 2017. DOI:10.1016/j.ejps.2017.01.032. 